\title{
Ingesta de Cuerpos Extraños Estudio Prospectivo Observacional. Conducta Terapéutica y Resultados
}

\author{
1Julio Trostchansky, ${ }^{2}$ Gabriel Masaferro
}

\section{ABSTRACTO}

El $80-90 \%$ de los cuerpos extraños ingeridos voluntaria o accidentalmente se evacuan espontáneamente, sin embargo se requiere de una endoscopia terapéutica en un $10-20 \%$ de los casos y de cirugía en menos del $1 \%$ de los mismos.

Objetivo: Analizar las características de los pacientes asistidos por ingesta de cuerpo extraño en el Departamento de Emergencia del Hospital Maciel y llegar al diseño de guías de actuación en el contexto de un equipo multidisciplinario.

Resultados: Nuestra serie constó de 31 consultas por ingesta de cuerpo extraño. El 52\% provenía de instituciones psiquiátricas y el $42 \%$ eran reclusos, un caso no pertenecía a ninguna de las 2 categorías. El 27,5\% de las consulta se habían producido en las primeras $24 \mathrm{hs}$ desde la ingesta, el $24 \%$ de las ingestas se habían producido con más de una semana desde la ingesta y el resto oscilaba entra ambos tiempos evolutivos En cuanto a la conducta, en 12 casos se otorgó el alta sin mediar conducta activa endoscópica y/o quirúrgica. Se realizó una FGC en el $64 \%$ de los casos en los cuales se constató radiológicamente la ingesta de un cuerpo extraño, siendo efectivamente terapéuticas para la extracción de objetos a nivel gastroduodenal en el $67 \%$ de las mismas. De las 28 consultas por ingesta de CE 6 pacientes (21\%) fueron operados. En 5 de ellos por falla de la extracción endoscópica, el restante se presentó como una peritonitis aguda. La ingesta de CE representa una patología de consulta frecuente en el Departamento de Emergencia. El manejo del mismo se realiza mediante un abordaje multidisciplinario que en el caso de nuestra serie mostró porcentajes de extracción endoscópica efectiva cercana al $70 \%$ comparable con otras series internacionales. El análisis de las características de la población analizada y el análisis de la evidencia científica publicada nos permite plantear un protocolo de actuación.

Palavras-chave: Cirugía, Cuerpos extraños, Prisiones.

How to cite this article: Trostchansky J, Masaferro G. Ingesta de Cuerpos Extraños Estudio Prospectivo Observacional. Conducta Terapéutica y Resultados. Panam J Trauma Crit Care Emerg Surg 2017;6(3):146-154.

Source of support: Nil

Conflict of interest: None

\footnotetext{
${ }^{1,2}$ Associate Professor

1,2Departamento de Cirugía, Hospital Maciel, Montevideo Uruguay
}

Corresponding Author: Julio Trostchansky, Associate Professor Departamento de Cirugía, Hospital Maciel, Montevideo, Uruguay Phone: +59894046621, e-mail: juliotrostchansky@gmail.com

\section{ABSTRACT}

About 80 to $90 \%$ of foreign bodies ingested voluntarily or accidentally are spontaneously ejected; however, a therapeutic endoscopy is required in 10 to $20 \%$ of cases and surgery in less than $1 \%$ of them.

Objective: To analyze the characteristics of assisted patients due to foreign body ingestion in the Department of Emergency of Maciel Hospital and the design of protocols of action in the context of a multidisciplinary team.

Results: Our series had 31 patients who came for consultation because of foreign body ingestion; $52 \%$ came from psychiatric care institutions, $42 \%$ were prisoners of state prison, and one case did not belong to any of the previous categories. About $27.5 \%$ of consultations occurred within the first 24 hours after ingestion, $24 \%$ of consultations in the Department of Emergency occurred after more than 1 week from ingestion, and the rest oscillated between both evolutionary times. Regarding the therapeutic behavior performed, in 12 cases it was decided to discharge the patient without active endoscopic conduct or surgery. A FGC was performed in $64 \%$ of cases in which the intake of the foreign body was found radiologically. The FGC was effectively therapeutic for the extraction of objects at the gastroduodenal level in $67 \%$ of them. Of the 28 visits to the Department of Emergency for foreign body ingestion, 6 patients $(21 \%)$ were operated. The reason for this surgery was the failure of endoscopic extraction in 5 of them, the remaining patient presented with acute peritonitis. Ingestion of foreign body represents a pathology of frequent consultation in the Department of Emergency. The management of this clinical situation is carried out through a multidisciplinary approach that, in the case of our series, showed percentages of effective endoscopic extraction close to $70 \%$, comparable with other international series. The analysis of the characteristics of the population studied in our series and the analysis of the published scientific evidence allow us to define a protocol of action of this type of patients in our hospital.

Keyword: Foreign body, Prisons, Surgery.

\section{INTRODUCCION}

El 80-90\% de los cuerpos extraños ingeridos voluntaria o accidentalmente se evacuan espontáneamente, ${ }^{1}$ sin embargo se requiere de una endoscopia terapéutica en un $10-20 \%$ de los casos y de cirugía en menos del 1\% de los mismos. ${ }^{2}$ En el caso del paciente privado de libertad este porcentaje de cirugías es francamente mayor cercano al $18 \%$ de los pacientes.

La ingesta de cuerpos extraños en un $80 \%$ de los casos se da deforma accidental en la población pediátrica. ${ }^{3}$ Sin embargo en adultos la ingesta accidental ocurre fundamentalmente en pacientes psiquiátricos o reclusos 
carcelarios. $^{2}$ En este último grupo predomina la ingesta de hojas de afeitar, pilas u otros objetos metálicos cortantes o con sectores afilados. ${ }^{1}$

En la población de pacientes que se atienden en el Hospital Maciel por ingesta de cuerpos extraños es clara la predominancia de la ingesta de "bombillas" como luego analizaremos. Instrumento éste metálico que forma parte del mate, bebida típica de nuestro país, de gran difusión entre la población y a la cual tiene fácil acceso la población carcelaria y de instituciones psiquiátricas. Sus características lo hacen propicio para la ingesta, dado que es cilíndrico-alargado, de diámetro máximo que oscila $1-2 \mathrm{~cm}$, no cortante y que en promedio mide entre $15 \mathrm{y}$ $20 \mathrm{~cm}$, en general ingieren fragmentos de las mismas.

Tal es la dimensión de este problema en nuestro Hospital y nuestro país, que si comparamos nuestro volumen de consultas con el que reportan las últimas series publicadas como la de Belfast, ${ }^{4}$ la diferencia es alarmante, como luego destacaremos. Ello nos obliga a estudiar nuestra realidad con el fin de poder establecer pautas diagnósticas y terapéuticas acordes a dicha problemática.

\section{OBJETIVO}

El objetivo del presente trabajo es:

- Analizar las características de los pacientes asistidos por ingesta de cuerpo extraño en el Departamento de Emergencia del Hospital Maciel

- Compararlo con la evidencia publicada

- El diseño de guías de actuación en el contexto de un equipo multidisciplinario.

\section{MATERIAL Y MÉTODOS}

Todos los pacientes durante el período comprendido entre el 1 de abril de 2010 al 31 de marzo del 2011 cuyo motivo de consulta fuera la ingesta intencional de cuerpo extraño fueron incluidos en el presente estudio. Los pacientes con ingesta accidental (huesos de pollo o espina de pescado) obstrucción por alimentos o ingesta intencional de sustancias narcóticas fueron excluidos. La información se recolectó en forma prospectiva durante el periodo mencionado. Para ello se diseño un formulario estandarizado y cerrado (Anexo al final del artículo) contemplando elementos tales como los datos patronímicos, endoscopías o laparotomías previas en referencia a dicho problema, la sintomatología, el tipo de material ingerido así como los estudios diagnósticos o terapéuticos solicitados y la conducta terapéutica tomada por el equipo actuante. En relación a todos los aspectos recabados en el protocolo no existía hasta el momento del estudio protocolos o guías de acción en nuestro Hospital referido al manejo de la ingesta de cuerpos extraños por lo que las acciones asistenciales se realizaban según decisión del equipo actuante.

Los pacientes fueron admitidos en el Departamento de Emergencia del Hospital Maciel y evaluados por un equipo multidisciplinario conformado por el médico internista, psiquiatra, cirujano y médico endoscopista.

Todos los pacientes dieron su consentimiento informado para la realización de los estudios realizados.

Las complicaciones fueron definidas como todo efecto adverso en la evolución del paciente durante su internación o que motivara su reingreso.

\section{RESULTADOS}

Nuestra serie constó de 31 consultas por ingesta de cuerpo extraño. De la cuales 28 resultaron en confirmación del motivo de consulta. En los tres restantes no se constataron imágenes radiológicas compatibles con el cuerpo extraño informado como ingerido.

El 52\% provenía de instituciones psiquiátricas y el 42\% eran reclusos, un caso no pertenecía a ninguna de las 2 categorías. El 27,5\% de las consulta se habían producido en las primeras $24 \mathrm{hs}$ desde la ingesta, el 24\% de las ingestas se habían producido con más de una semana desde la ingesta y el resto oscilaba entra ambos tiempos evolutivos.

La localización del cuerpo extraño, naturaleza del mismo, sintomatología inicial, edad (media) y sexo de la población estudiada se muestra en la Tabla 1.

A todos se les realizo radiografía orientada al síntoma y de ser asintomático se realizó enfoque torácico y abdominal. En el 96,5\% de los casos en los que se confirmó la ingesta, el cuerpo extraño se localizó a nivel abdominal, el caso restante fue torácico.

Tabla 1: Caracteristicas de la muestra

\begin{tabular}{lllll}
\hline Topografia & Torax & Abdomen & Dudoso & Sin objeto \\
& 1 & 28 & 0 & 3 \\
Tipo objeto (casos) & Bombilla & Cuchillo o similar & Otros & Sin cuerpo extraño \\
& 14 & 7 & 7 & 3 \\
Corto-punzantes & $\mathrm{Si}$ & No & Sin cuerpo extraño & \\
Sintomas & 7 & 21 & Dolor tx & 1 \\
& Asintomaticos & Dolor abdominal & & \\
Sexo & 19 & $11(1$ perotonitis) & & \\
& Masculino & Femenino & & \\
\hline
\end{tabular}




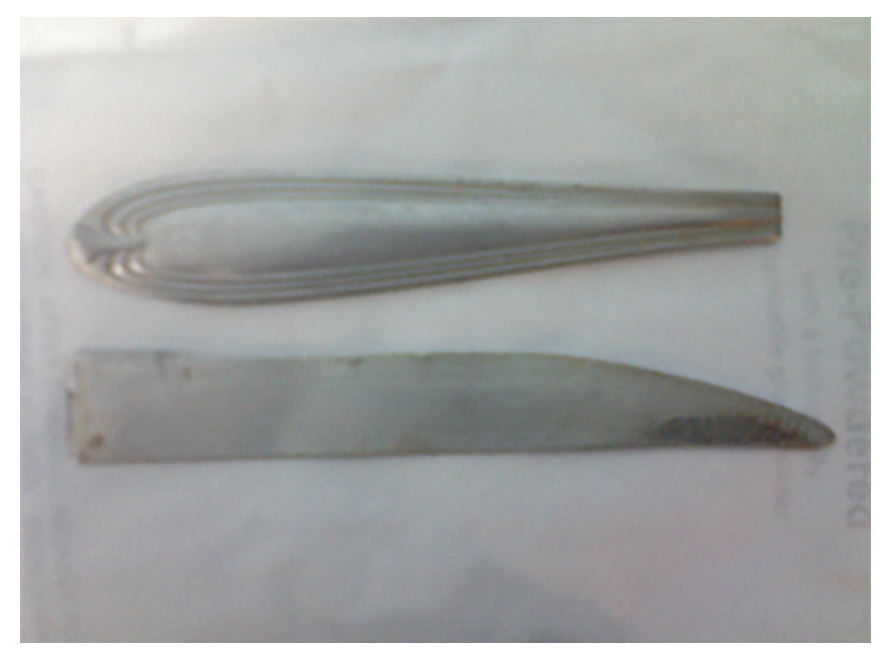

Figura 1: Trozos de cuchillos extraídos endoscópicamente

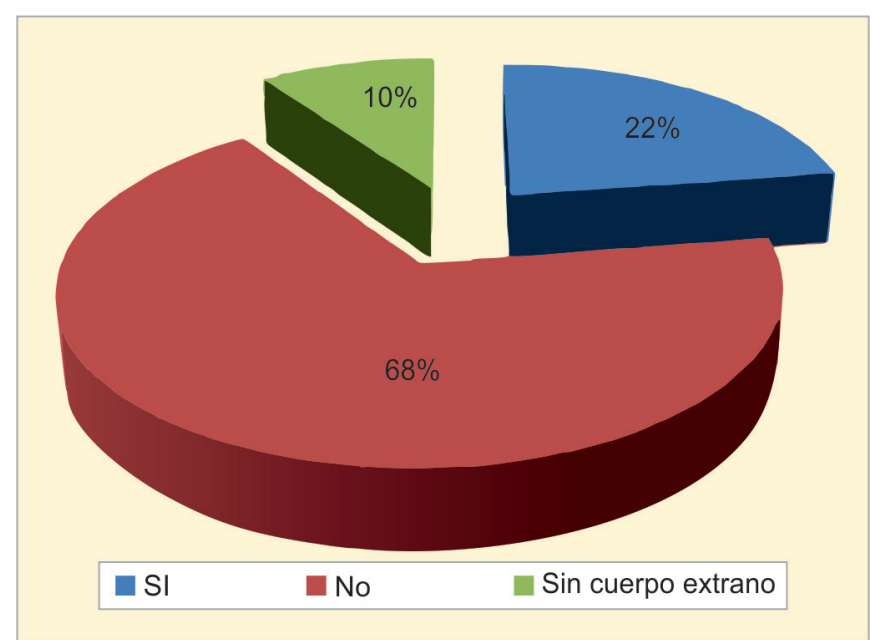

Grafica 2: hallazgo endoscópico de ingesta de objeto corto punzante

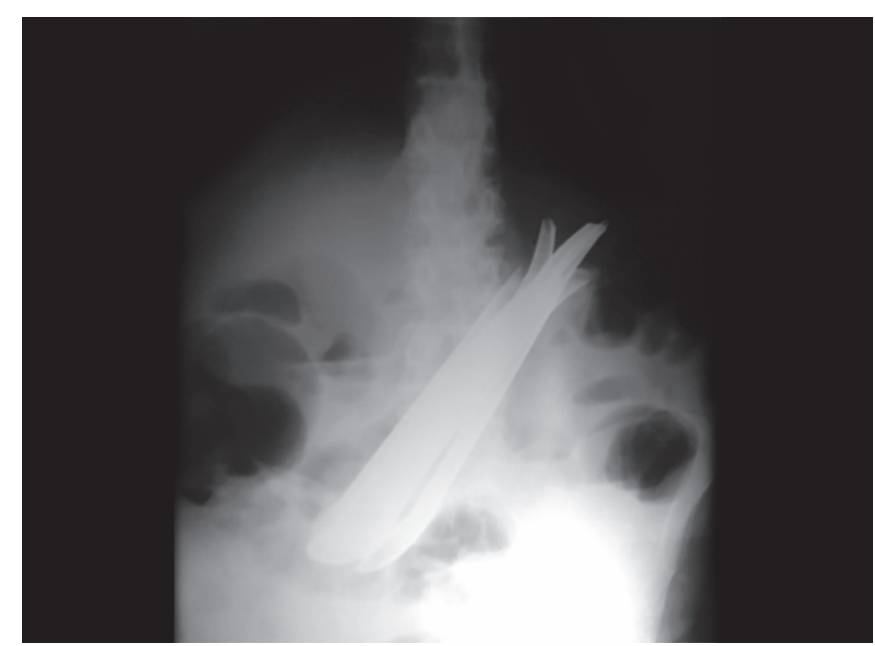

Figura 3: Paciente que había ingerido 4 objetos corto punzantes diferentes

En cuanto a las características de lo ingerido el 75\% eran objetos no corto-punzantes y dentro de éstos el $66 \%$ eran bombillas (Figura 1), 25\% eran corto-punzantes (Gráfica 1 y 2) (Figura 2 y 3 y 4). El $42 \%$ presentaba 1 CE,

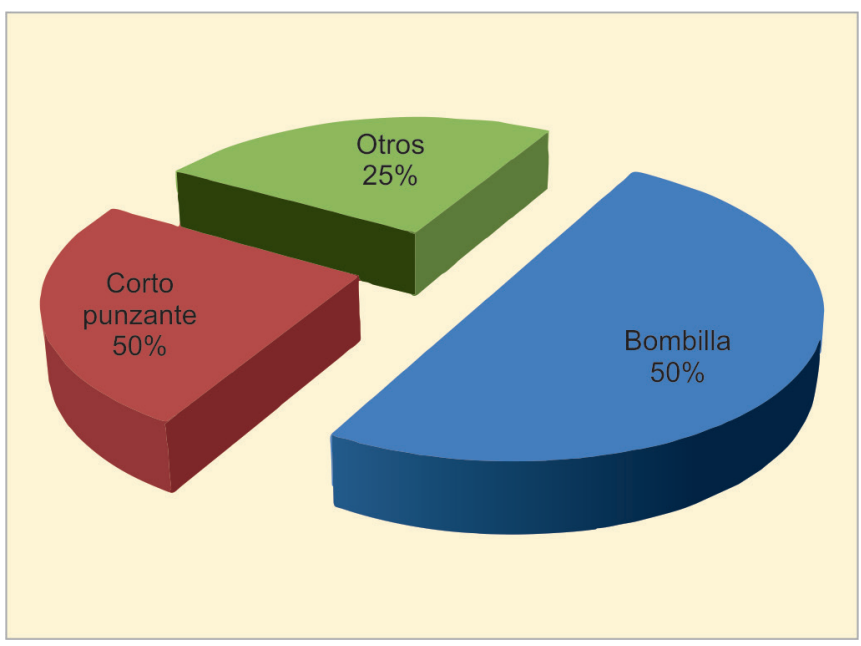

Grafica 1: Objeto ingerido

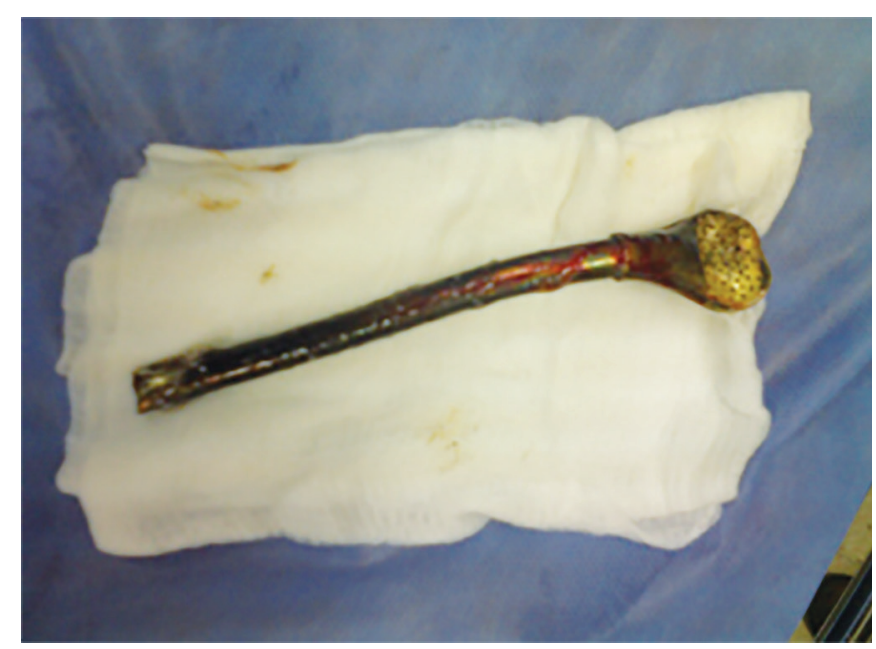

Figura 2: Bombilla extraída endoscópicamente (La bombilla es un elemento con el que se toma el Mate una infusión típica en Uruguay)

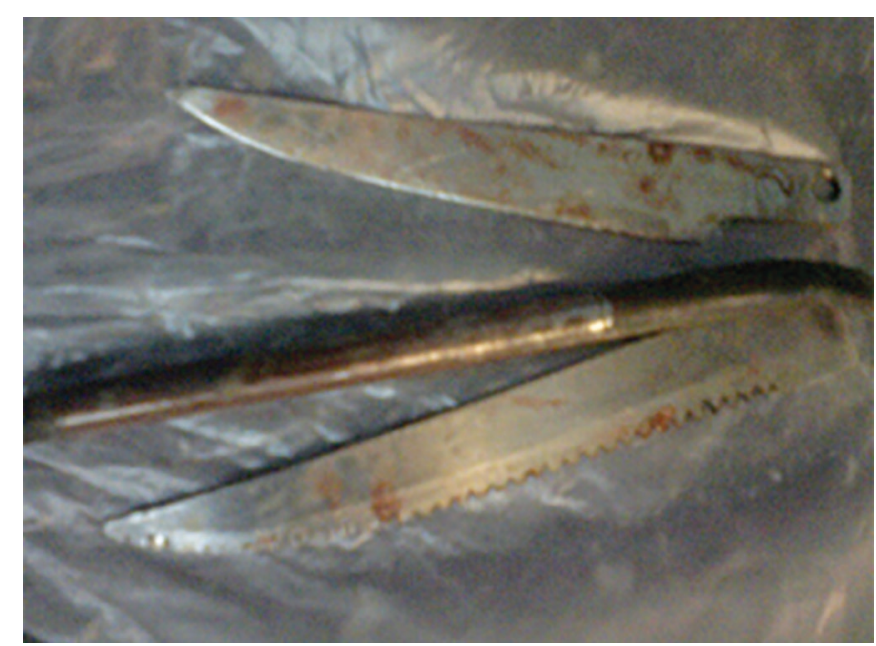

Figura 4: Objetos extraídos del paciente citado en la figura 3

el $28,5 \%$ presentaba $2 \mathrm{CE}$ ingeridos, el $14,5 \%$ presentaba $3 \mathrm{CE}$ ingeridos, el $4 \%$ presentaba $4 \mathrm{CE}$ ingeridos y el $11 \%$ presentaba 5 CE ingeridos (Gráfica 3). El 89\% de los objetos ingeridos tenían un largo mayor a $5 \mathrm{~cm}$ (Gráfica 4). 


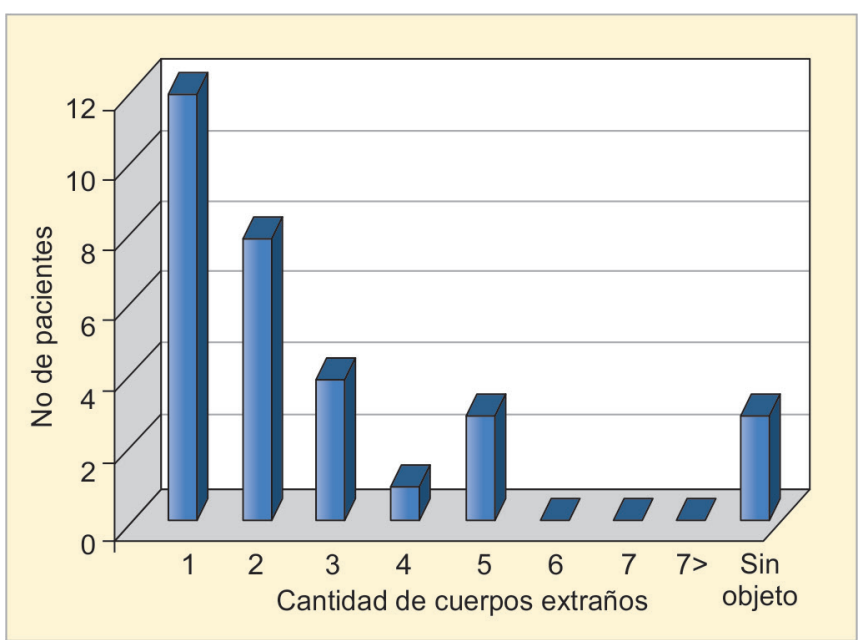

Grafica 3. Número de cuerpos extraños por paciente

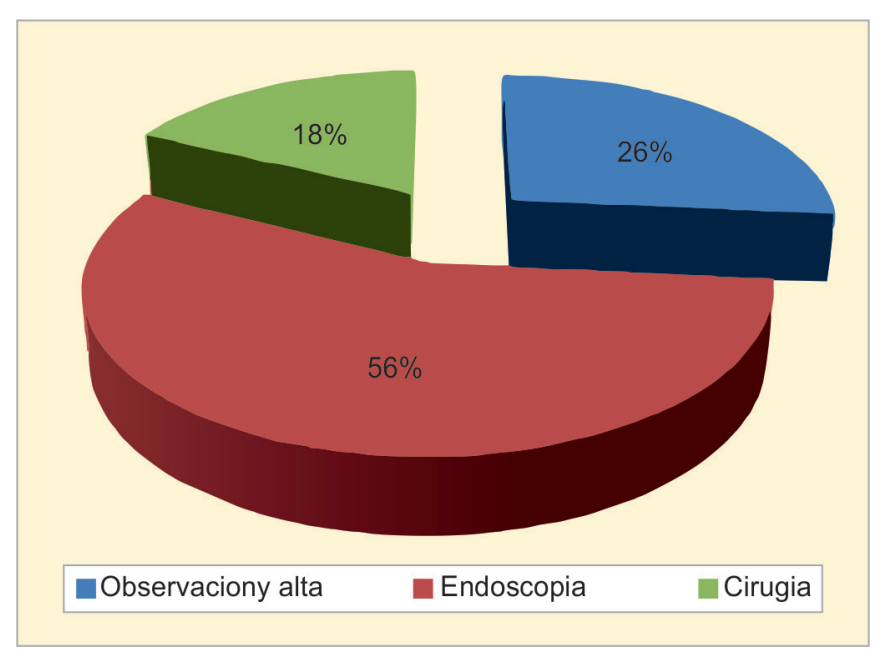

Grafica 5: Conducta terapéutica

En cuanto a la conducta, en 12 casos se otorgó el alta sin mediar conducta activa endoscópica y/o quirúrgica (Gráfica 5) incluidos los pacientes en los cuales no se encontraron imágenes radiológicas patológicas. En 3 casos el CE se encontraba a nivel gastroduodenal siendo todos elementos no cortantes. Todos eran mayores de $5 \mathrm{~cm}$. En dos de los tres casos el manejo conservador falló ya que requirieron cirugía en un caso y FGC terapéutica posterior al momento de la re-consulta. Los restantes casos fueron 4 casos a nivel del intestino delgado, uno de ellos cortante, y 2 a nivel colónico, uno de ellos cortante. (Gráfica 6 y 7). No hubo re-consulta de estos pacientes en nuestra Institución.

Se realizó una FGC en el $64 \%$ de los casos en los cuales se constató radiológicamente la ingesta de un cuerpo extraño, siendo efectivamente terapéuticas para la extracción de objetos a nivel gastroduodenal en el $67 \%$ de las mismas (12 casos). En 2 casos el paciente tenía en forma sincrónica cuerpos extraños a nivel del colon y / o intestino delgado por lo que puede considerarse que, la efectividad

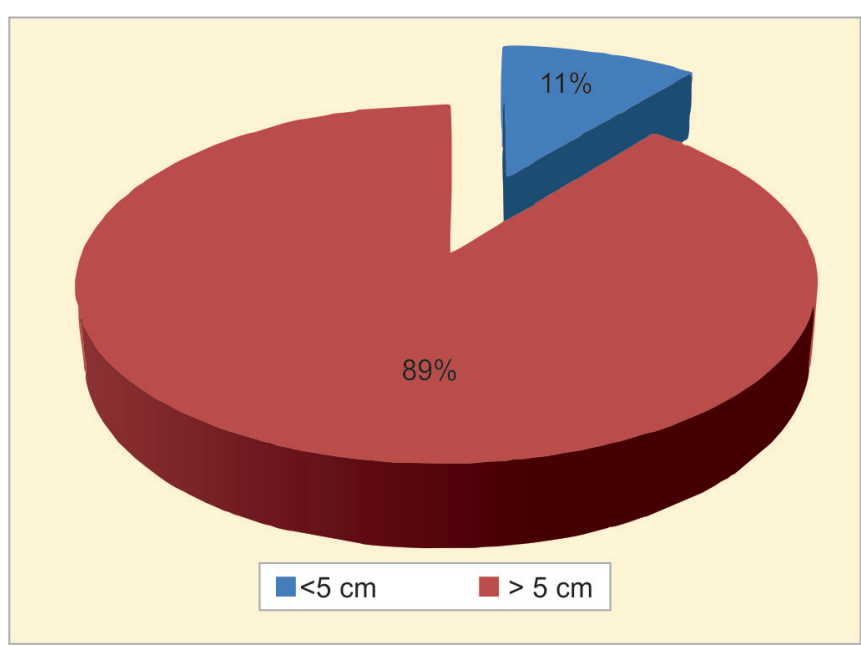

Grafica 4: Longitud del objeto ingerido

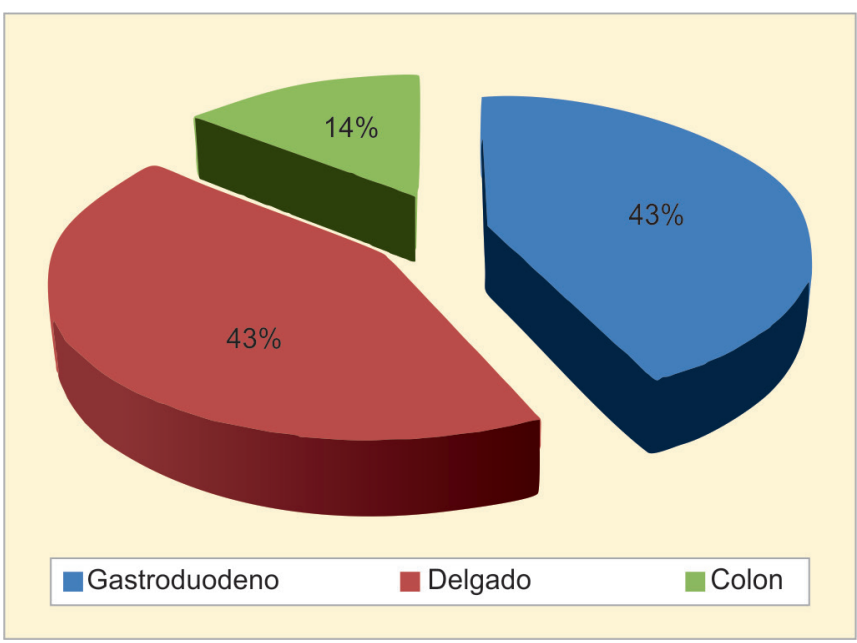

Grafica 6: Topografía del cuerpo extraño en pacientes con conducta terapéutica expectante

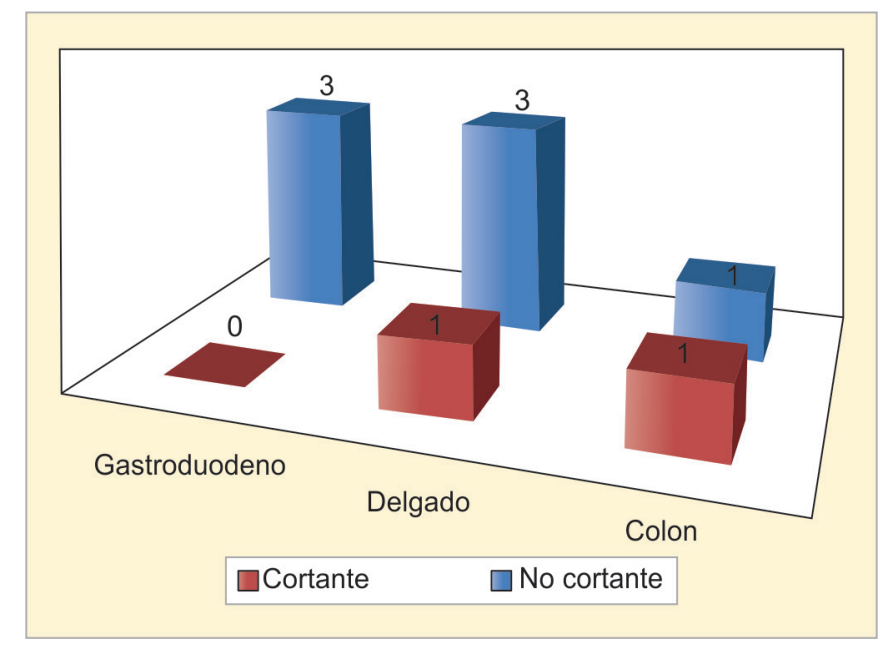

Grafica 7: Características del objeto en pacientes con conducta terapéutica expectante

real en la resolución global de la ingesta de cuerpos extraños fue del 53\% (Gráfica 8). De todas maneras hasta el momento de la finalización del estudio ninguno de los dos pacientes mencionados había reconsultado. 


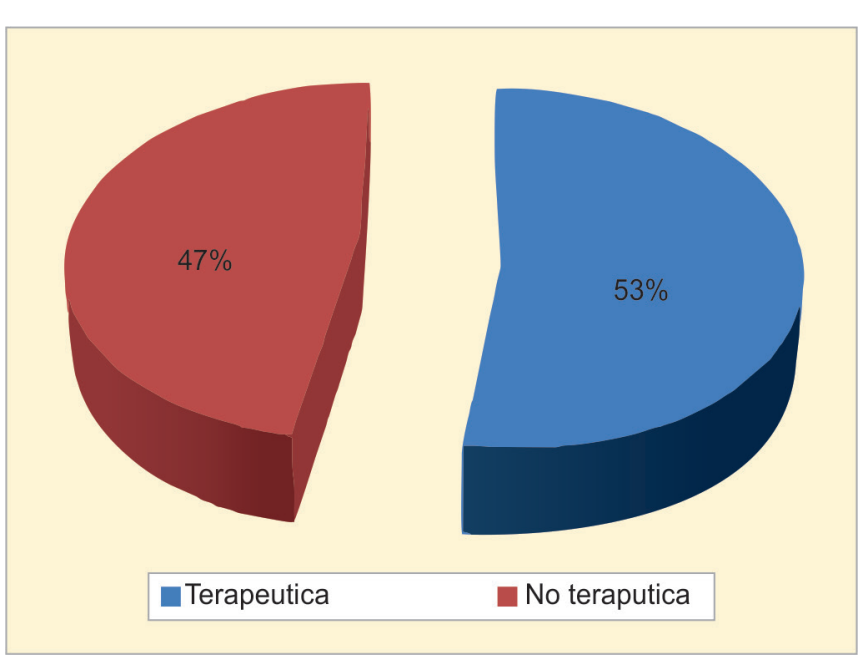

Grafica 8: Resultado terapéutico de la endoscopía

Las endoscopías no terapéuticas para la extracción de $\mathrm{CE}$ a nivel gastroduodenal fueron 6, sus características se detallan en la Tabla 2. Destacamos que en 5 de estos casos los objetos superaban los $10 \mathrm{~cm}$ de largo. Como contrapartida de las 12 endoscopias terapéuticas en 7 los $\mathrm{CE}$ eran mayores de $10 \mathrm{~cm}$. De las 28 consultas por ingesta de CE 6 pacientes (21\%) fueron operados. En 5 de ellos por falla de la extracción endoscópica, el restante se presentó como una peritonitis aguda (Gráfica 9).Los procedimientos quirúrgicos realizados se enumeran en la Gráfica 10.

\section{DISCUSIÓN}

En los Estados Unidos se registran un promedio de 1500 muertes al año a causa de la ingesta de cuerpos extraños. ${ }^{5}$ Al contrario de lo publicado por la serie del 2008 de Belfast ${ }^{4}$ que en un plazo de 10 años reportaron 11 casos, otros autores como Blaho y cols ${ }^{6}$ demostraron una alta incidencia de ingesta de cuerpos extraños en personal carcelario al igual que Lee y cols que reportaron en 6,5 años 52 consultas en 33 pacientes de una población carcelaria de Korea. ${ }^{7}$

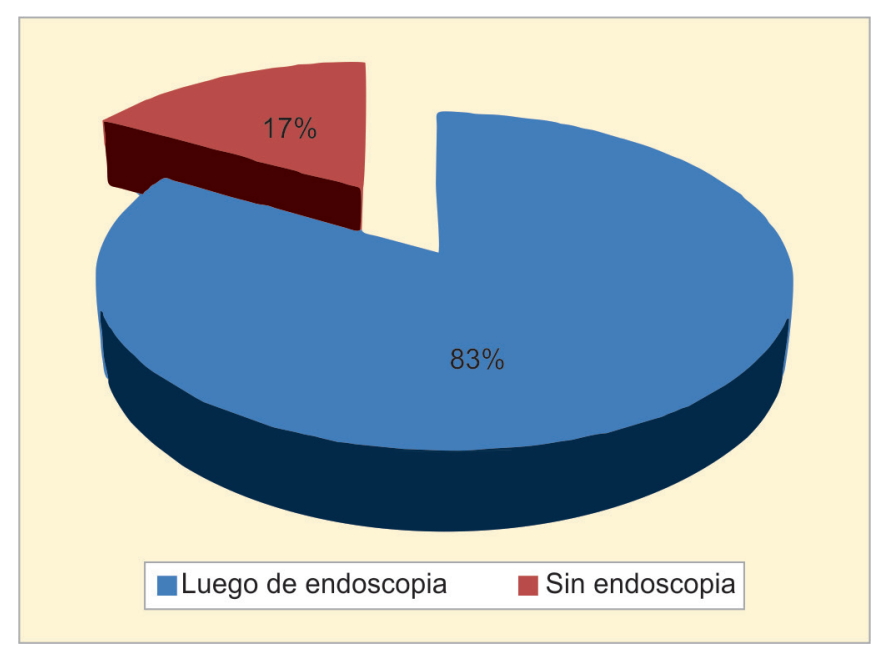

Grafica 9: Oportunidad de la Indicación de cirugía
Tabla 2. Endoscopias no terapeuticas. caracteristicas $Y$ conducta

\begin{tabular}{llll}
\hline & Cortante & Largo $(\mathrm{cm})$ & Conducta \\
\hline Caso 1 & No & 10 & Cirugia \\
Caso 2 & No & 20 & Cirugia \\
Caso 3 & No & 15 & Cirugia \\
Caso 4 & No & 12 & Cirugia \\
Caso 5 & Si & 10 & Cirugia \\
Caso 6 & No & 7 & Alta \\
\hline
\end{tabular}

Es de destacar que la mayoría de los objetos metálicos, exceptuando el aluminio, son radio-opacos. Esto último es desconocido por la mayoría de las personas, incluyendo radiólogos. ${ }^{8}$ También lo son los huesos de animales y el vidrio. La mayoría de los plásticos, maderas o espinas de pescado no son radio-opacos, por el contrario pueden verse como zonas radio-lúcidas. Esto es de fundamental importancia para no descartar la ingesta de cuerpos extraños a pesar de que sean negativas las radiografías, siendo por ello muy importante la realización de un interrogatorio exhaustivo sobre las características del objeto ingerido.

En cuanto a la presentación clínica en su mayoría son asintomáticos. Existen presentaciones clínicas bajo forma de complicación como el sangrado digestivo ${ }^{6}$ o la perforación intestinal como ocurrió en uno de los pacientes de nuestro estudio.

La impactación de los cuerpos extraños ocurre a nivel de las estrecheces fisiológicas o angulaciones. Ello ocurre a nivel crico-faríngeo, del arco aórtico, del bronquio fuente izquierdo, del esfínter esofágico inferior, del píloro, de la válvula ileo-cecal o el ano. La impactación de un cuerpo extraño que dura más que 24 horas se asocia con el aumento en la incidencia de complicaciones. ${ }^{10}$ La ocurrencia de apendicitis aguda por impactación de un cuerpo extraño es una complicación rara aunque interesante. ${ }^{11}$

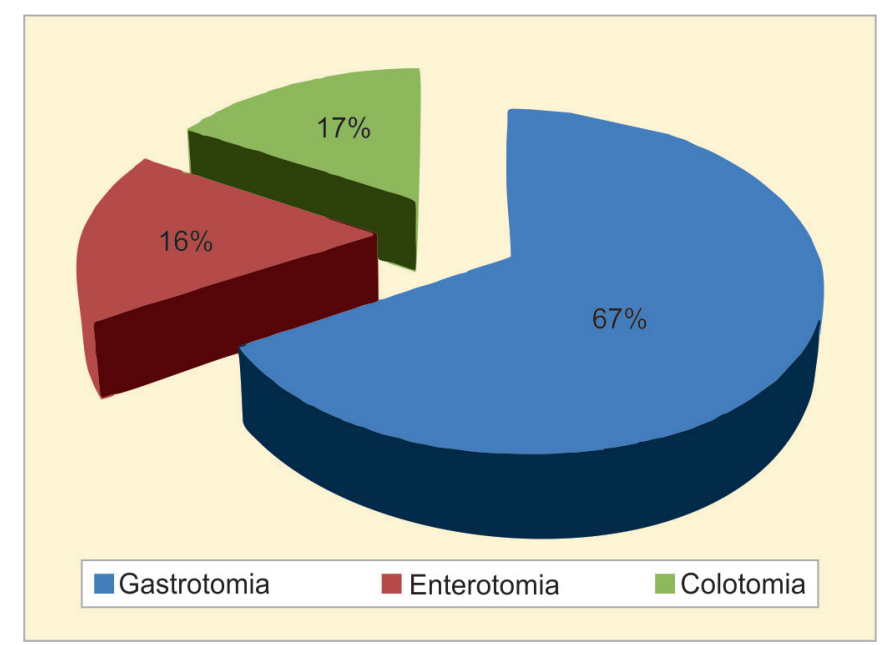

Grafica 10: Tipo de cirugía realizada 
Los objetos metálicos, tales como agujas u objetos alargados como los huesos de pescado, huesos de pollo, y palillos de dientes son los cuerpos extraños que más frecuentes se han reportado como causa de perforación. ${ }^{12}$ Los sitios más comunes de la perforación son el esófago inferior y el íleon terminal. ${ }^{2,13}$

Pueden presentarse clínicamente con disfagia, odinofagia o dolor precordial ${ }^{14}$ si presentan impactación a nivel esofágico, los 2 primeros son los síntomas más frecuentemente reportados. ${ }^{15}$ También vómitos ${ }^{16} \mathrm{o}$ regurgitaciones. ${ }^{17}$ Dentro del mismo esófago los sitios de impactación están influenciados no solo por las estrecheces anatómicas conocidas sino también por la edad, ${ }^{17,18}$ el tipo de cuero extraño, ${ }^{19,20}$ así como la duración de la ingesta y condiciones patológicas particulares como ser estricturas, estenosis o fístulas previas. Los signos de alarma de la impactación de un cuerpo extraño a nivel esofágico o en vía aérea incluyen estridor, ${ }^{21}$ sialorrea $^{22} \mathrm{u}$ otros síntomas respiratorios como tos y asfixia. ${ }^{16}$ El enfisema subcutáneo es un elemento orientador de perforación esofágica y obliga a una correcta valoración imagenológica como se discute más adelante.

En más de 2.000 revisiones de casos de ingestión de cuerpo extraño, sólo en 25 casos (1\%) se objetivó la perforación esofágica por huesos de pescado o pollo, de estos solo dos casos desarrollaron una fístula aortoesofágica como complicación fatal. ${ }^{23}$

En el 2007 Hunt y cols reportaron un caso de fistula aorto-esofágica tardía a los 10 días de la ingesta de 2 hojas de afeitar, los autores presuponen la perforación esofágica con absceso intermediario y fistula posterior dado que en el momento de la muerte de la paciente la radiografía mostraba la hojas de afeitar a nivel del colon derecho. ${ }^{24}$ Esta presentación coincide con lo que se describe como la tríada de Chiari, que incluye dolor torácico, hemorragia centinela, un período de tiempo asintomático seguido de exanguinación fatal. ${ }^{25}$

A pesar de la perforación de víscera hueca algunos pacientes permanecen asintomáticos, como reportan Hashimonai y cols en un paciente con perforación duodenal por agujas. ${ }^{26}$

Desde el punto de vista diagnóstico una radiografía de cuello, tórax y abdomen debe ser realizada al ingreso y orientada según la clínica del paciente. De presentarse asintomático recomendamos solicitarlas todas. En el caso de nuestro estudio ello nos permitió diagnosticar y suponer la topografía en el $90 \%$ de los pacientes así como suponer su ausencia en el 10\% restante.

Asimismo la ubicación radiológica nos puede indicar la necesidad de otros estudios o su contraindicación. Algunos autores recomiendan no realizar una endoscopia digestiva si hay evidencia radiológica o sospecha clínica de lesión esofágica, estos pacientes deberían ser valorados con Tomografía Computada (TC). ${ }^{27}$ El elemento mas útil para el diagnostico tomográfico de perforación es la presencia de aire extra-luminal. $^{28}$

La utilización de la TC tiene una sensibilidad de 100\% y es la primera opción para el diagnóstico de cuerpo extraño a nivel del esófago superior si se sospecha que el mismo no será visible en la radiografía. ${ }^{29}$ La alta resolución y sensibilidad de la TC hacen de éste un estudio de alto rendimiento diagnóstico para detectar cuerpos extraños. ${ }^{30}$ La TC de abdomen o tórax puede ser útil si un área de opacidad o inusual radio-lucidez se encuentra en la radiografía. ${ }^{31}$ Debido a las características de los CE ingeridos por la población de estudio no fue necesario la utilización de la TAC como método diagnóstico.

Tomados en forma global la ingesta de cuerpos extraños que llegan al tracto gastrointestinal progresarán espontáneamente en un porcentaje aproximadamente del $80 \%$. El 10 a 20\% requerirán de maniobras no quirúrgicas y solo el $1 \%$ necesitará de una cirugía. La observación es la conducta preferida en aquellas ingestas de menos de 24 hs de cuerpos extraños no peligrosos, pequeños y que se presentan asintomáticos.

En el caso de los objetos no cortantes de menos de $2 \mathrm{~cm}$ que se encuentren a nivel gástrico la conducta es preferentemente expectante con radiología semanal y de forma ambulatoria. En el lumen gástrico los objetos son frecuentemente retenidos por largos períodos sin presentar consecuencia. ${ }^{32} \mathrm{Si}$ el objeto no cortante permanece en el estómago puede retrasarse la endoscopía 1-2 meses para darle oportunidad al pasaje espontáneo del objeto 2,33,34. No obstante, la mayoría de los CE de nuestro estudio eran de gran tamaño, muchos de ellos cortantes y casi la mitad se presentó con síntomas. Ello además en una población como la carcelaria o con patología psiquiátrica que se caracteriza por tener un porcentaje de cirugías mayor que la población general. En nuestro estudio la tasa de cirugía fue del $21 \%$.

Los avances recientes en el manejo de la endoscopía flexible hacen de la misma una opción diagnóstica y terapéutica de primera línea en todos los pacientes con historia de ingesta de $\mathrm{CE}$ con las características de nuestra población.

Nuestro estudio presenta una particularidad que hace a la región del Cono Sur. El 66\% de los objetos no corto punzantes ingeridos fueron bombillas para la infusión mate. Ello explica el tamaño encontrado en muchos de los mismos. En general, los cuerpos extraños superiores a 2-2,5 cm de tamaño no entran en el canal pilórico y los superiores a 6-10 $\mathrm{cm}$ de longitud no progresan a través de la curvatura del duodeno. ${ }^{1,2,35}$ Es por ello que deben de ser retirados endoscópicamente o quirúrgicamente. En nuestra serie el $89 \%$ de los objetos ingeridos tenían un largo mayor a $5 \mathrm{~cm}$. De nuestra serie destacamos que los CE localizados a nivel gastroduodenal todos eran mayores a $5 \mathrm{~cm}$, por lo 
que asumiendo la experiencia publicada no progresarían a través del tubo digestivo. Ello quedó demostrado en dos de los tres pacientes con CE gastroduodenal manejados con conducta expectante. En los 2 casos se dio la re-consulta requiriendo de una FGC y de una cirugía. Claramente la observación y el alta de estos pacientes sin intervención alguna en la consulta no resuelve su patología y no debería de alentarse. La no progresión de dichos cuerpos extraños amerita la cirugía si la FGC no es efectiva.

La recuperación endoscópica de objetos afilados, como de hojas de afeitar, sujetapapeles y agujas que se presenten en el esófago se debe realizar con urgencia. ${ }^{1,2}$ Del mismo modo si el objeto avanza hasta el estómago o el duodeno, los intentos de recuperación endoscópica deben llevarse a cabo de inmediato, ya que el riesgo de perforación en la válvula ileocecal es de aproximadamente 35\%. 1,2,12

En nuestro estudio la tasa de éxito en la extracción endoscópica de CE a nivel gastroduodenal fue del $67 \%$ lo que se acerca a lo reportado en otras series. La falla en la extracción endoscópica se dio en un caso por no contar con el material adecuado (sobre tubo endoscópico) para extraer el objeto cortante y en los restantes el objeto no cortante era mayor a $10 \mathrm{~cm}$, menos en un caso que media $7 \mathrm{~cm}$ pero de todas formas no se pudo extraer. En este ultimo caso se otorgo el alta lo cual es un claro error de procedimiento dado que como se mencionó no progresaría por su tamaño a nivel distal, este paciente no reconsultó hasta el final del estudio. Si bien como se hizo referencia previamente la falla de la endoscopía en la extracción de CE se presentó en la mayoría da los casos con cuerpos extraños mayores a $10 \mathrm{~cm}$ (5 en 6) destacamos que en los casos donde la endoscopía fue terapéutica, 12 casos, en 7 de ellos el largo del CE superaba los $10 \mathrm{~cm}$. Por lo que si bien el largo del objeto superior a $10 \mathrm{~cm}$ determinó la falla de la endoscopía en varios casos, este pudiera no ser un predictor confiable de ello dado que en más del 50\% de las endoscopías terapéuticas el CE presentaba más de $10 \mathrm{~cm}$. Para dilucidar este punto se debería plantear un estudio que cuente con un $\mathrm{N}$ más amplio.

Si el objeto ha pasado más allá del duodeno y es no cortante y no supera los $5 \mathrm{~cm}$ se puede hacer un seguimiento 2 veces por semana observando su evolución. Si es punzante independientemente de su tamaño, el paciente debe ser monitoreado con radiografías diarias y permanecer bajo observación estricta. La intervención quirúrgica puede ser necesaria si un objeto punzante no progresa radiológicamente después de 72 horas. Es necesario realizar una laparotomía de urgencia si el paciente desarrolla síntomas que sugieran una complicación. ${ }^{1,2} \mathrm{De}$ los CE localizados a nivel del intestino delgado ninguno superaba los $5 \mathrm{~cm}$ de largo pero uno de los casos era por CE cortante lo cual configura una indicación formal de ingreso y observación, con cirugía de no progresar en
72 hs, ninguna de etas 2 conductas fue realizada constituyendo un claro error de actuación. De los CE localizados a nivel colónico las 2 consultas fueron del mismo paciente y con CE cortante por lo cual la conducta de alta sin mediar terapéutica no solo fue un claro error de actuación sino que se repitió en 2 oportunidades. Debemos desalentar enfáticamente esta conducta. La conducta debió de ser la de realizar el ingreso hospitalario del paciente, radiografía diaria y cirugía de no progresar en $72 \mathrm{hs}$.

La indicación de cirugía surge frente a la falla de la extracción endoscópica o la aparición de complicaciones. En las perforaciones esofágicas la cirugía es la mejor opción, ${ }^{36}$ con diferentes tácticas aplicables, pero también están descritas conductas conservadoras no quirúrgicas, ${ }^{37}$ y endoscópicas. ${ }^{38}$

De presentares elementos de irritación peritoneal la cirugía es mandatoria. La táctica quirúrgica no difiere de la que se utiliza en la reparación de una perforación intestinal por otra etiología.

\section{CONCLUSION}

La ingesta de CE representa una patología de consulta frecuente en el Departamento de Emergencia. En un año hubo casi 3 consultas por mes por esta patología.

El manejo del mismo se realiza mediante un abordaje multidisciplinario que en el caso de nuestra serie mostró porcentajes de extracción endoscópica efectiva cercana al $70 \%$ comparable con otras series internacionales.

Sin embargo, hemos detectado fallas en la toma de decisiones terapéuticas que pueden comprometer la evolución favorable de los pacientes. El análisis de las características de la población analizada y el análisis de la evidencia científica publicada nos permite plantear un protocolo de actuación según el "estado del arte" de dicha patología y adaptado a la realidad de nuestro medio hospitalario que es en todo aplicable a la mayoría de los hospitales de nuestro país.

\section{PROTOCOLO DE ACTUACIÓN ANTE LA INGESTA DE CE}

Paciente que consulta por ingesta de CE:

- Examen físico:

- Signos o síntomas de perforación del tubo digestivo $\rightarrow$ CIRUGÍA SEGÚN TOPOGRAFÍA DE LA PERFORACION (Clínico-IMAGENOLÓGICO)

- Sin síntomas de perforación de tubo digestivo $\rightarrow R X$ y actuar según topografía y características del CE según los criterios que se mencionan a continuación.

\section{CE ESOFAGICO}

- CORTANTE: ENDOSCOPÍA URGENTE

- NO CORTANTE 
- Objeto impactado o dificultad respiratoria ENDOSCOPÍA

- Pila de botón - ENDOSCOPÍA

- Estabilidad hemodinámica y sin compromiso respiratorio y no pila de botón :

ENDOSCOPÍA OBLIGADA

- Moneda (localización proximal a nivel esofágico, la no tolerancia de líquidos vía oral o las sucesivas radiografías positivas que evidencien la falta de progresión distal): MANEJO ESPECTANTE o ENDOSCOPÍA o Ambulatorio.

\section{CE GASTRICO}

- CORTANTE: ENDOSCOPÍA URGENTE

- NO CORTANTE

- bateria DE bOTON ENDOSCOPÍA SI $\rightarrow$ PERMANECE + DE 48HS Y DIAMETRO > 2CM. EXPECTANTE EN EL RESTO

- $<2 \mathrm{~cm}$ EXPECTANTE, AMBULATORIO CON RX SEMANAL y ENDOSCOPÍA SI NO PROGRESA EN 30 DÍAS

- > 2CM ENDOSCOPÍA y CIRUGía (FALLO ENDOSCÓPICO).

\section{CE INTESTINAL}

- CORTANTE INGRESAR/Radiografía DIARIA/CIRUGIA SI NO PROGRESA EN 72HS O COMPLICACION

- NO CORTANTE SEGUIMIENTO RADIOLOGICO 2 VECES POR SEMANA. Cirugía si hay complicación.

\section{BIBLIOGRAFIA}

1. Pavlidis TE, Marakis GN, Triantafyllou A, Psarras K, Kontoulis TM, Sakantamis AK. Management of ingested foreign bodies: how justifable is a waiting policy? Internet J Surg 2007:9(1).

2. Eisen GM, Baron TH, Dominitz JA, Feigel DO, Goldstein JL, Johanson JF et al; American Society for Gastrointestinal Endoscopy. Guideline for the management of ingested foreign bodies. Gastrointest Endosc 2002;55(7):802-806.

3. Barros JL, Caballero A Jr., Rueda JC, Monturiol JM. Foreign body ingestion: management of 167 cases. World J Surgery 1991;15(6):783-788.

4. Bisharat M, O'Donnell ME, Gibson N, Mitchell M, Refsum SR, Carey PD, et al. Foreign Body Ingestion in Prisoners - The Belfast Experience. Ulster Med J 2008;77(2):110-114.

5. Uyemura MC. Foreign body ingestion in children. Am Fam Physician 2005;72:287-291.

6. Blaho KE, Merigian KS, Winbery SL, Park LJ, Cockrell M. Foreign body ingestion in the Emergency Department: case reports and review of treatment. J Emerg Med 1998;16(1):21-26.

7. Lee TH, et al. Foreign Objects in Korean Prisoners. The Korean Journal of Internal Medicine 2007;22:275-278.

8. Eggli KD, Potter BM, Garcia V, Altman RP, Breckbill DL. Delayed diagnosis of esophageal perforation by aluminum foreign bodies. Pediatr Radiol 1986;16:511-513.
9. Lai ATY, et al. Risk factors predicting the development of complications after foreign body ingestión. British Journal of Surgery 2003;90:1531-1535.

10. Chaves DM, Ishioka S, Felix VN, et al. Removal of a foreign body from the upper gastrointestinal tract with a flexible endoscope: a prospective study. Endoscopy 2004;36:887-892.

11. Balch CM, Silver D. Foreign bodies in the appendix. Arch Surg 1971;102:14-20.

12. Maglinte DDT, Taylor SD, Ng AC. Gastrointestinal perforation by chicken bones. Radiology 1979;130:597-599.

13. Murshid KR, Khairy GE. Laparoscopic removal of a foreign body from the intestine. J R Coll Surg Edinb 1998;43(2): 109-111.

14. Nijhawan S, Shimpi L, Mathur A, Mathur V, Roop Rai R. Management of ingested foreign bodies in upper gastrointestinal tract: report on 170 patients. Indian J Gastroenterol 2003;22(2):46-48.

15. Sittitrai P, Pattarasakulchai T, Tapatiwong H. Esophageal foreign bodies. J Med Assoc Thai 2000;83(12):1514-1518.

16. Diaz GA, Valledor L, Seda F. Foreign bodies from the upperaerodigestive tract of children in Puerto Rico. Bol Asoc Med P R 2000;92(9-12):124-129.

17. Al-Qudah A, DaradkehS, Abu-Khalaf M. Esophageal foreign bodies. Eur J Cardiothorac Surg 1998;13(5):494-498.

18. Baraka A, Bikhazi G. Oesophageal foreign bodies. Br Med J 1975;1(5957):561-563.

19. Higo R, Matsumoto Y, Ichimura K, Kaga K. Foreign bodies in the aerodigestive tract in pediatric patients. Auris Nasus Larynx 2003;30(4):397-401.

20. Giordano A, Adams G, Boies L Jr, Meyerhoff W. Current management of esophageal foreign bodies. Arch Otolaryngol 1981;107(4):249-51.

21. Janik JE, Janik JS. Magill forceps extraction of upper esophageal coins. J Pediatr Surg 2003;38(2):227-9

22. Khan MA, Hameed A, Choudhry AJ. Management of foreign bodies in the esophagus. J Coll hysicians Surg Pak 2004;14(4): 218-220.

23. Nandi P, Ong GB. Foreign body in the oesophagus: review of 2394 cases. Br J Surg 1978;65:5-9.

24. Hunt I, et al. European Journal of Cardio-thoracic Surgery 31(2007):946-948.

25. Chiari H. Uber fremdkorperverletzung des oesophagus mitaortenperfora-tion (in German). Berl Klin Wschr 1914;51:7-9.

26. Hashmonai M, Kaufman T, Schramek A. Silent perforations of the stomach and duodenum by needles. Arch Surg 1978;113:1406-1409.

27. Low WK, Tay HN. Management of ingested foreign bodies. In: Hwang NC, Ooi L, editors. Acute Surgical Management. Singapore: World Scientific 2004:171-187.

28. White CS, et al. Esophageal perforation: CT findings. Am J Roentgenol 1993;160:767-770.

29. Marco De Lucas E, Sadaba P, Lastra Garcia-Baron P, et al. Value of helical computed tomography in the management of upper esophageal foreign bodies. Acta Radiol 2004;45: 369-374.

30. Santos SA, Alberto SCF, Cruz E, et al. Hepatic abscess induced by foreign body: case report and literature review. World J Gastroenterol 2007;13:1466-1470.

31. Berger PE, Kuhn JP, Kuhns LR. Computed tomography and the occult tracheobroncheal foreign body. Radiology 1980; 134:133-135. 
32. Zuloaga J, Olivera J, Candia A, Arias V, De la Fuente A. [Ingestion of foreign bodies in a prison population; surgical treatment] Spanish. Rev Esp Enferm Apar Dig 1987;72:617-621.

33. Sinnatamby CS, Editor Last's Anatomy: Regional and Applied. 10th ed. London: Churchill Livingstone: 2003. p. 241-256.

34. Samad L, et al. Button batery ingestión: Hazards of esophageal impactation. J Pediatr Surg 1999;34:1527-1531.

35. Litovitz TL. Battery ingestions: product accessibility and clinical course. Pediatrics 1985;75(3):469-476.
36. Namasivayam S. Button battery ingestion: a solution to a management dilemma. Pediatr Surg Int 1999;15(5):383-384.

37. Kao LS, Nguyen T, Dominitz J, Teicher HL, Kearney DJ. Modification of a latex glove for the safe endoscopic removal of a sharp gastric foreign body. Gastrointest Endosc 2000; 52 : 127-129.

38. Gavin CWK, et al. 980 Letter to the Editor. Successful Management of an Iatrogenically-ingested Sharp Foreign Body. Annals Academy of Medicine 2008;37:980-981. 